\section{Public Health, Mental Health and Primary Care Differences in Urban and Rural Counties in a System of Care for Children with Special Healthcare Needs}

\section{Abstract}

Background: Collaboration among partners in a health system of care (SOC) has many benefits. In large and complex systems, the role of public health, mental health, and primary care play critical roles in the provision of care. How these systems vary across urban and rural settings is understudied. Understanding of perceived roles and ongoing collaboration across these sectors in urban and rural communities is needed.

Methods: We assessed geographic and sector of care differences among members of a SOC providing care to CHSNC. In this study, dyadic data $(n=698)$ from a 2013 statewide SOC for CSHCN were analyzed to explore the relationships between sectors (mental health, public health and primary care) and geographic settings (urban, rural, mixed).

Results: The majority of partnerships were reported among urban partners $(n=484)$, followed by mixed $(n=136)$ and rural $(n=78)$. Significant variation was found in frequency $(p<0.001)$ and level of involvement by geographic setting $(p<0.001)$. Resource contribution, frequency of interaction, involvement, reliability, geographic setting, and mission congruence significantly differed across sectors.

Conclusions: This research indicates there are opportunities to strengthen primary care and public health relationships as well as leverage limited but valued mental health resources to further improve the care provided to CSHCN.

Keywords: Primary care, Mental health, Public health, Healthcare, Social networks, Partnerships

\section{Danielle M. Varda', Rose Hardy², Amanda Millar ${ }^{3}$ and Ayelet Talmi ${ }^{3}$}

1 School of Public Affairs University of Colorado Denver, Denver, USA

2 Colorado School of Public Health, University of Colorado Denver, USA

3Colorado School of Medicine, University of Colorado Denver, USA

\section{Corresponding author: Varda DM}

ફdanielle.varda@ucdenver.edu

PhD, Associate Professor, University of Colorado Denver, School of Public Affairs, 1380 Lawrence St., Ste. 500 Denver, CO80204, USA.

Tel: 3033152129

Citation: Varda DM, Hardy R, Millar A, et al. Public Health, Mental Health and Primary Care Differences in Urban and Rural Counties in a System of Care for Children with Special Healthcare Needs. Health Syst Policy Res. 2016, 3:3

Received: June 28, 2015; Accepted: July 26, 2016; Published: July 29, 2016

\section{Background}

Collaboration among partners in a system of care (SOC) has many benefits. In large and complex systems, the role of public health, mental health, and primary care play critical roles in the provision of care. How these systems vary across urban and rural settings is understudied. Understanding of perceived roles and ongoing collaboration across these sectors in urban and rural communities increases service coordination and quality of care and leverages limited available resources.

In the United States, an estimated 11.7 million children are identified as having special health care needs [1]. As defined by the Maternal and Child Health Bureau, these children "have or are at increased risk for a chronic physical, developmental, behavioral, or emotional condition and who also require health and related services of a type or amount beyond that required by children generally" [2]. Moreover, the chronic conditions that children with special health care needs (CSHCN) often experience require many forms of health care expertise and technology that extends far beyond their childhoods [3]. Even under the best of circumstances, families of CSHCN must navigate complex systems comprised of multiple organizations and providers across various sectors (i.e., mental health, public health, and primary care) in order to access basic services from qualified professionals. To add to the complexity, these services vary in terms of their 
service delivery and billing across various geographic boundaries. Ensuring comprehensive, coordinated, efficient and effective health care services and service delivery is thus paramount to both families and providers [4,5]. How factors such as geography and collaboration among sectors varies is less clear, including where such factors strengthen or hinder coordination.

Assessing how healthcare delivery networks function for CSHCN is important to families, providers, and other health care service partners. Well-managed, coordinated SOCs have the potential to successfully increase service coordination and responsiveness to patient and family needs [6]. This potential for success are amplified if efforts to improve systems' functioning better integrate the role of public health in coordination with Primary Care Networks (PCNs) and the manner in which these networks operate.

In this paper we address the questions:

1. What are the interactions among members of SOCs that are characterized as urban, rural, and mixed?

2. What are interactions among mental health, primary care, and public health organizations involved in coordinated services for CSHCN?

\section{Geographic differences among interactions in a SOC for CSHCN}

SOCs may differ by geographic setting, which can exacerbate or mediate health care delivery challenges. Urban systems of care serve larger populations, have more available and accessible resources, and provide more services through official organizations like public health departments and primary care providers. Alternatively, rural systems of care serve smaller populations, have fewer available resources, and more services are provided by faith-based and non-health related partners and organizations [7]. Access to services is beneficial when stakeholders are acquainted with these services and utilize them efficiently. In cases where services are unknown or difficult to access, underutilization and poor efficiency in health care service delivery occurs. While rural networks may have fewer resources, they may also be more familiar with their partners and existing services. Currently, there is limited information on SOCs that span geographic lines. Understanding how urban and rural partners collaborate and perceive each other provides insight into effective healthcare delivery.

\section{Sector differences among interactions in a system of care for CSHCN}

In addition to these geographic differences, there may also be differences in the level of involvement and scope of services provided by different sectors, or domains, of care. Primary care, public health, and mental health partners play vital roles in the lives of CSHCN families [8]. Understanding how partners interact and perceive one another when caring for CSHCN provides insight into health care delivery. Additionally as debate continues over the provision of clinical services by public health departments, understanding the current relationships and scope of these sectors of care can provide valuable information [9-11]. Gaining an understanding of the nuances of geographic setting and sector of care in a system of care influences successful partnerships can be used to further improve care provision.

\section{Methods}

This analysis is part of a larger social network analysis study (collected in 2013) of a statewide SOC for CSHCN in Colorado [8]. The PARNTER survey (www.partnertool.net) was used and distributed to system stakeholders in Colorado that cared for CSHCN. The survey is validated and uses social network analysis methodology to collect data from respondents on who they interact with to coordinate care for CSHCN, what they do as partners for coordination, and the quality and perceptions of those partnerships. Measures collected in the survey are listed in Table 1, and include frequency of interaction, perceptions of power and influence, resource contribution, level of involvement, reliability, mission congruence, and communication. (scaled as "none", "a small amount", "a fair amount" or "a great deal").

The study population included stakeholders in the SOC for CSHCN in Colorado. The unit of analysis in this study was organizational dyads, meaning a respondent representing an organization and organizations with which they reported working with to care for CSHCN. In the survey, respondents were asked to identify members of the SOC serving these CSHCN families, describe the quantity and quality of exchange between organizations, and provide assessments of the trust and value of those members. The goal was to assess the connectivity of organizations in this SOC in Colorado. The focus of this analysis (completed in 2015) was to assess how geographic setting differences and SOC affected those perceptions of trust and value. This study was reviewed and approved by the Colorado Institutional Review Board.

These dyadic data were categorized by "sector" of nominated organizations, the organization the respondent indicated they worked with when caring for CSHCN. The three sectors included in this analysis were primary care, public health, and mental health partners. Dyadic data were also categorized as urban, rural, or mixed geographic setting. These categorizations were based on the Health Resources and Services Administration's definition of metro and non-metro counties [12]. When both

Table 1 PARTNER Survey Questions, by Topic.

\begin{tabular}{|c|c|}
\hline $\begin{array}{c}\text { Relationship Factor } \\
\text { Frequency }\end{array}$ & $\begin{array}{c}\text { Definition } \\
\text { Prequency of interaction with organization } \\
\text { Perceived power/influence of organization } \\
\text { as prominent member of the community, or } \\
\text { agent of change }\end{array}$ \\
\hline Resource Contribution & $\begin{array}{r}\text { Perceived value of organization's resource } \\
\text { contribution (money, food, physical space, } \\
\text { staff, data, etc) }\end{array}$ \\
\hline Revel of Involvement & $\begin{array}{r}\text { Perceived value of organization's level of } \\
\text { involvement }\end{array}$ \\
\hline Mission Congruence & $\begin{array}{r}\text { Perceived level of reliability in organization's } \\
\text { follow through on commitments }\end{array}$ \\
\hline Communication & $\begin{array}{r}\text { Perceived level of open communication with } \\
\text { organization }\end{array}$ \\
\hline
\end{tabular}


partners were based in an urban county they were categorized as urban. When both partners were based in rural counties then they were categorized as rural. When one partner was in an urban county and the other in a rural county, then they were classified as mixed.

Descriptive chi square analysis was performed to assess differences based on geographic setting and sector. A p-value less than 0.05 was considered statistically significant. The relationship measures assessed included frequency of interaction and perceptions of power/influence, resource contribution, level of involvement, reliability, mission congruence, and communication [13].

\section{Results}

This survey was distributed to 295 system stakeholders and obtained a $34 \%$ response rate (101 organizations responded to the survey). Each responding organization nominated organizations that they interacted with to coordinate care for $\mathrm{CSHCN}$, yielding partnerships among 432 total organizations and 698 dyads. Of the 698 organizational partnerships, 484 were classified as urban, 78 were classified as rural, and 136 were classified as mixed.

The percentage of primary care, public health, mental health, and other organizations composing each geographic setting are presented in Figure 1. In viewing these partnerships by sector of care, urban settings have a variety of organizations involved in the care of CSHCN. In rural settings, public health partners make up $22 \%$ of the organizations involved in the care of CSHCN and their families and primary care partners make up $15 \%$ of their partners $(\chi 2=30.32, p<0.001)$. Although the proportion of mental health organizations involved in rural networks is small, $2.5 \%$, they compose $6.7 \%$ of organizations that cross geographic lines $(p<0.001)$. This suggests that rural $\mathrm{CSHCN}$ may receive mental health services from urban partners.
The geographic network measures are displayed in Figure 2. In visualizing all dyads together at the whole network level by geographic setting, we find that urban networks tend to be more centralized while the rural networks are smaller and have fewer connections among all the members. The rural networks had a higher density, measured as the number of partnerships that exist of all that possibly could exist, implying that rural systems of care maximize their potential ties more than urban or mixed systems by activating more of the available partnerships. The rural networks degree centralization score, a measure of the degree to which a few organizations have the most ties (centralization) rather than a more equal distribution of ties among organizations (decentralization) was lower indicating these systems are more decentralized than urban or mixed systems. However, rural networks had high transitivity scores, measured as the number of closed "triples" - any three organizations and the number that are closed, or all three organizations are connected, implying that these networks have more relationships were their partners know one another and are connected than urban or mixed systems of care.

Reported frequency of interaction and perceived level of involvement by partners were found to be significantly different by geographic setting, with $42.2 \%$ of urban partnerships, $24.7 \%$ of rural partnerships, and $22.1 \%$ of mixed partnerships reporting interacting at least once a month $(\chi 2=28.19, p<0.001)$. In terms of perceived levels of involvement, $38.3 \%$ of urban partnerships, $25.6 \%$ of rural partnerships, and $21.6 \%$ of mixed partnerships perceived a great deal of involvement from their partners when caring for these children $(\chi 2=17.09, p=0.009)$.

In order to understand the nuances of different sectors of care, we examined primary care, public health, and mental health organizations by geographic setting. Figure 3 demonstrates differences across these sectors and geographic settings.

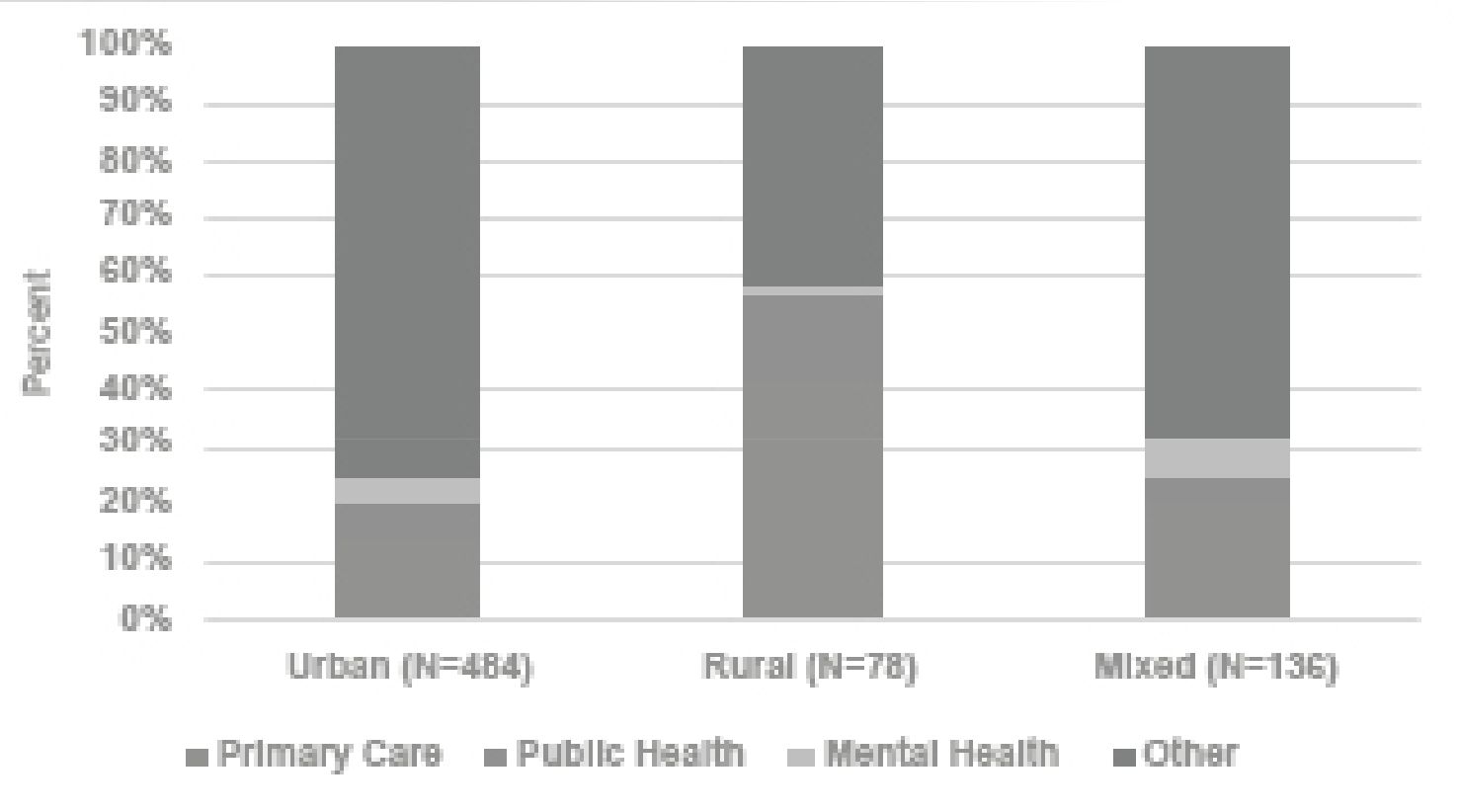

Figure 1 Percentage of Each Sector of Organizations by Geographic Setting. 


\begin{tabular}{|l|l|ll|}
\hline Network Type: & Urban & Mixed & Rural \\
\hline & & & \\
& & & \\
& & & \\
Density & 0.006 & 0.008 & 0.112 \\
\hline Centralization & 0.118 & 0.22 & 0.014 \\
Transitivity & 0.137 & 0.00 & 0.36 \\
\hline
\end{tabular}

The line drawn in each figure shows any rating of a sector below "a fair amount". Urban dyads rated primary care and public health partners highest, while rural dyads were rated highest their public health partners, and mixed dyads rated highest for mental health.

Among primary care partners, several relationship measures differed significantly by geographic setting. These included frequency of interaction, perceived resource contribution, level of involvement, reliability, and mission congruence. While $84.4 \%$ of urban respondents perceived at least a fair amount of resource contribution from their urban primary care partners, only $50 \%$ of rural respondents felt similarly about their rural primary care partners and $59.3 \%$ of respondents felt that same level of resource contribution from their primary care partner crossing geographic lines $(x 2=13.27, p=0.039)$. Although $57.4 \%$ of urban partnerships reported interacting at least once a month, only 9.1\% of rural partnerships and $25.9 \%$ of mixed partnerships reported such frequent interactions $(\chi 2=19.24, p=0.004)$.

Among public health partners, resource contribution was found to be statistically different by geographic setting. Half of urban partnerships perceived resource contribution of their partners as greatly valuable in achieving the overall goals of their collaborative while $41.2 \%$ of rural partnerships and $28.6 \%$ of mixed partnerships felt similarly $(\chi 2=13.75, p=0.03)$. Relationship measures on perceptions of trust and value were found to be high in rural and urban settings suggesting public health partners were well perceived. More than $90 \%$ of respondents across all geographic settings reported that their public health partners were highly reliable.

Among mental health partners, only perceived reliability was found to differ significantly by geographic setting. Although
$18.8 \%$ of urban respondents reported trusting urban partners a great deal, $50 \%$ of rural respondents reported a great deal trust in rural mental health partners. That percentage increased when mental health partners crossed geographic lines, with $77.8 \%$ of respondents reporting a great deal of trust $(\chi 2=8.75, p=0.022)$. In general it appeared that mental health partners crossing geographic lines were viewed more positively than in either urban or rural settings.

\section{Discussion}

\section{Main findings of this study}

Examining public health, primary care, and mental health sectors by geographic setting yielded important finding about the roles and relationships among sectors in systems of care for CSHCN. A number of key findings can inform how these SOCs might benefit from this deeper understanding of the barriers, roles, and considerations for strengthening such a system. These are discussed below.

Primary care partners are more valued and trusted among their urban partners, while rural and mixed partners reported less positive perceptions.

Perceptions of trust of primary care partners were relatively high across geographic settings while perceptions of value of primary care partners was surprisingly low in rural settings. The majority of mean primary care partner scores on perceptions of the value of partnerships in rural and mixed geographic settings are considered below the benchmark as positive, in relation to over 500 comparable networks in the PARTNER tool dataset $[14,15]$. This may indicate that primary care partners in SOCs are not currently as valued as other mental health and public health partners in rural and mixed settings, giving way for better 


\section{Primary Care}

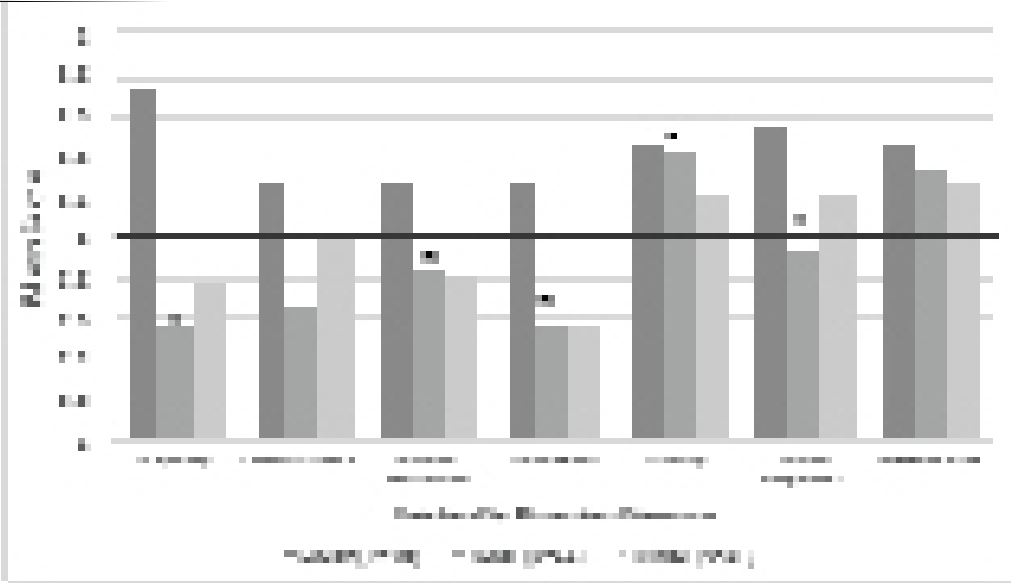

Public Health

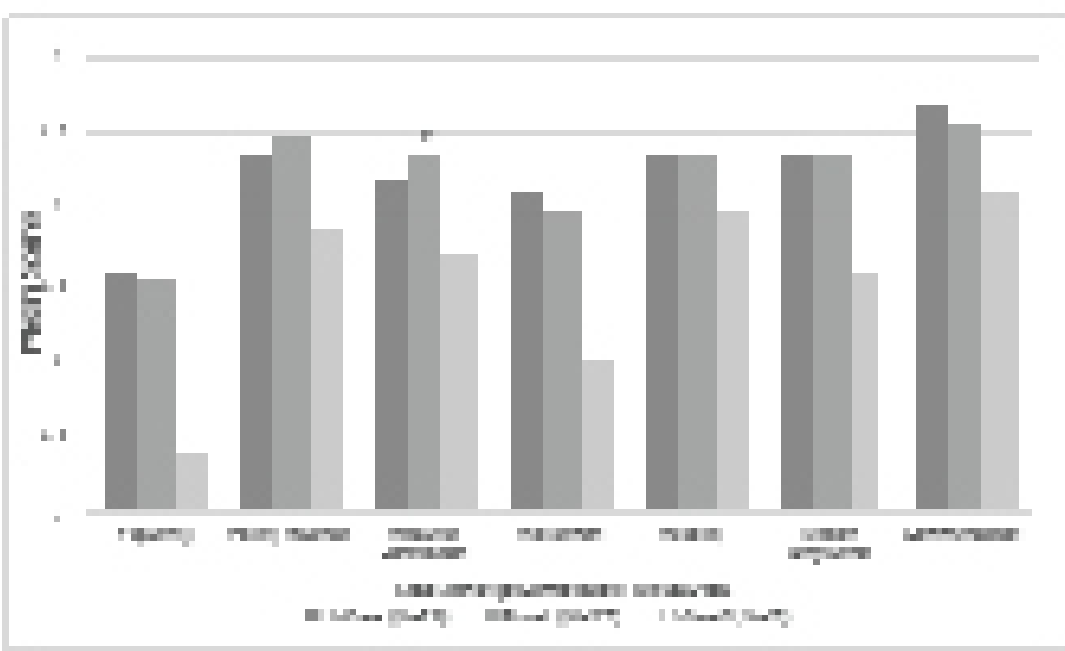

Mental Health.

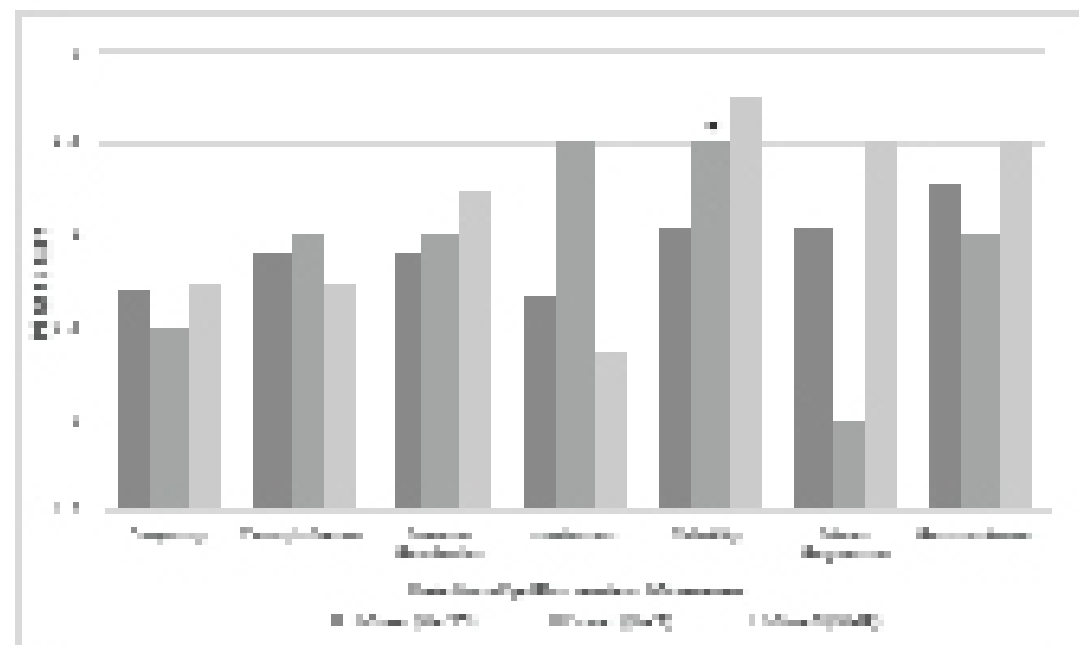

Figure 3 Mean Relationship Dimension Scores by Geographic Dyad Type. 
recognition and definition of the role that primary care plays in these partnerships that involve rural partners. Further, work may need to be done in rural and mixed settings to improve perceptions of primary care partners and strengthen their relevance and salience as partners within systems of care for CSHCN. Public health partners were often reported as having less interaction in the SOCs, however these interactions are highly valued and trusted.

Less frequent interactions were reported among rural health partners, most likely due to smaller population sizes and fewer CSHCN in these rural communities as compared to urban communities. However, respondents across geographic settings rated partners in the public health sector highly on dimensions of trust and value. The larger proportion of public health partners in rural settings suggests that there is room for public health organizations to play a larger role in the care of CSHCN in rural communities. Although the IOM recently recommended public health departments move away from the provision of clinical services, public health partners may need to play a more active role in the systems of care in rural communities [11]. For example, public health professionals may serve as connectors, identifying the needs of families with CSHCN and providing referrals to necessary resources in the community. Public health professionals are often uniquely aware of available resources and work actively to partner with community-based organizations to enhance access to these resources. Public health departments also convene community partners to address population health matters. As such, the public health sector plays a pivotal role in creating comprehensive, coordinated, family-centered systems of care, particularly in rural areas where other service sectors may be limited in their presence and scope.

Mental Health partners are less active in SOCs, however play highly valued and trusted roles for partnerships that cross geographic lines.

While mental health partners in rural networks were scarce, they are better represented in networks that crossed geographic lines. Perceptions of mental health partners increase when partners are a mix of urban and rural organizations with generally high perceptions of these organizations overall. While evidence suggests there are fewer mental health services in rural communities [16-18], much of the rural mental health care provision may be done by urban-based organizations. The high perception scores for mental health partners crossing geographic lines suggests that these mixed partnerships can be leveraged to improve rural mental health care provision.

\section{What is already known on this topic}

Characterizing roles and relationships among service sector partners can be used to inform decisions about policy, funding, and resource allocation that is optimized based on geographic setting. Currently, in this study, the findings tend to reflect assumptions about the way SOCs are functioning. For example, we saw that primary care providers are more active in urban partnerships and tend to be more valued in those settings, however they are both less active and valued in rural partnerships. They are however, highly trusted across geographic partner types. Public health partners have established highly valued and trusted partnerships across geographic settings, yet tend to have minimal involvement in these SOCs. Finally, mental health partners seem to play an important role in bridging across geographic lines, as they were rated as highly valued and trusted in mixed geographic partnerships (albeit with perceptions of less value as partners in urban settings).

\section{What this study adds}

These findings have important policy and practice implications. For example, public health partners have a lot of capital already developed in their highly trusted and value reputations in SOCs. Taking advantage of this and becoming more actively involved in coordination of SOC, as leaders, facilitators, and conduits of systems building is a role that can leverage these qualities. In other research, these authors came to a similar conclusion when looking at the potential role for public health through a systems lens [19]. In that work, they found that public health could play an important role in coordinating systems efforts (that is, coordinating care through the multiple stakeholders and providers families must navigate in their care), as the bridge between families and the more complex system.

Another example includes expanding access to mental health services in rural settings via partnerships between rural and urban partners. While rural settings lack sufficient resources for mental health services to adequately address SOCs needs, this may be best addressed by increasing cross-geographic partnerships with urban areas that are often more resource-rich than by attempting to attract, build, and retain rural mental health services. These findings show that when this approach has been implemented, better perceptions of the partnerships result.

Finally, primary care providers are scarce and valuable resources throughout SOCs in rural settings, reported in these data as less active/present in rural settings but perceived as very reliable and communicative. In order to bridge the gap in primary care provider availability in rural settings, programs promoting alternatives such as telemedicine could improve both the availability and perceptions of value by rural partners for PCPs.

\section{Limitations of this Study}

More work can be done to understand the impact of geography and sector type on SOCs. This research is limited in its availability of in-depth, qualitative data from members of these SOCs. Those nuances that can better inform policy and practice were not captured in these data, however ongoing work to address the gaps in these systems are underway and informed by this work. Current efforts include data collection that further explores how mental health, primary care, and public health can work harmoniously to coordinate care for $\mathrm{CSHCN}$.

\section{Acknowledgements}

The authors want to acknowledge the Colorado Trust for their funding of the WONDER babies partnership, the subject of this research. 


\section{References}

1 Newacheck PW, Kim SE (2005) A national profile of health care utilization and expenditures for children with special health care needs. Arch Pediatr Adolesc Med 159: 10-17.

2 McPherson M, Arango P, Fox H, Lauver C, McManus M, et al. (1998) A new definition of children with special health care needs. Pediatrics 102: 137-140.

3 Browne JV, Deloian B (2007) Infants and toddlers with special health care needs in Colorado: Identification, description, needs, and recommendations. White paper by the Center for Family and Infant Interaction and Colorado Department of Public Health and Environment.

4 Homer CJ, Klatka K, Romm D, Kuhlthau K, Bloom S, et al. (2008) A review of the evidence for the medical home for children with special health care needs. Pediatrics 122: e922-e937.

5 Huang ZJ, Kogan MD, Yu SM, Strickland B (2005) Delayed or forgone care among children with special health care needs: An analysis of the 2001 National Survey of Children with Special Health Care Needs. Ambul Pediatr 5: $60-67$.

6 Spencer SA, Blau GM, Mallery CJ (2010) Family-driven care in America: More than a good idea. J Can Acad Child Adolesc Psychiatry 19: 176-181.

7 Beatty K, Harris JK, Barnes PA (2010) The role of interorganizational partnerships in health services provision among rural, suburban, and urban local health departments. J Rural Health 26: 248-258.

8 Varda DM, Talmi A (2013) A Patient-Centered Approach for Evaluating Public Health Roles within Systems of Care for Children with Special Healthcare Needs. Front Public Health Serv Syst Res 2: 7.

9 Hale NL, Smith M, Hardin J, Brock-Martin A (2015) Rural Populations and Early Periodic Screening, Diagnosis, and Treatment Services:
Challenges and Opportunities for Local Public Health Departments. Am J Public Health 105: S330-S336.

10 Hsuan C, Rodriguez HP (2014) The adoption and discontinuation of clinical services by local health departments. Am J Public Health 104: 124-133.

11 Institute of Medicine (US) (2003) Committee on Assuring the Health of the Public in the 21st Century. The Future of the Public's Health in the 21st Century. National Academy Press.

12 (2010) Health Resources and Services Administration, Office of Rura Health Policy. List of rural counties and designated eligible census tracts in metropolitan counties.

13 Varda DM, Chandra A, Stern SA, Lurie N (2008) Core dimensions of connectivity in public health collaboratives. J Public Health Manag Pract 14: E1-E7.

14 Retrum J, Chapman C, Varda D (2013) Implications of network structure on public health collaboratives. Health Educ Behav 40(IS): 13S-23S.

15 Varda DM, Retrum JH (2015) Collaborative performance as a function of network members' perceptions of success. Public Perform Manag 38: 632-653.

16 Human J, Wasem C (1991) Rural mental health in America. Am Psychol 46: 232.

17 Kelleher KJ, Taylor JL, Rickert VI (1992) Mental health services for rural children and adolescents. Clin Psychol Rev 12: 841-852.

18 Yuen EJ, Gerdes JL, Gonzales JJ (1996) Patterns of rural mental health care: An exploratory study. Gen Hosp Psychiatry 18: 14-21.

19 Varda DM, Chandra A, Stern SA, Lurie N (2008) Core dimensions of connectivity in public health collaboratives. Journal of Public Health Management and Practice 14: E1-E7. 